\title{
Dural-based atypical teratoid/rhabdoid tumor in an adult: DNA methylation profiling as a tool for the diagnosis
}

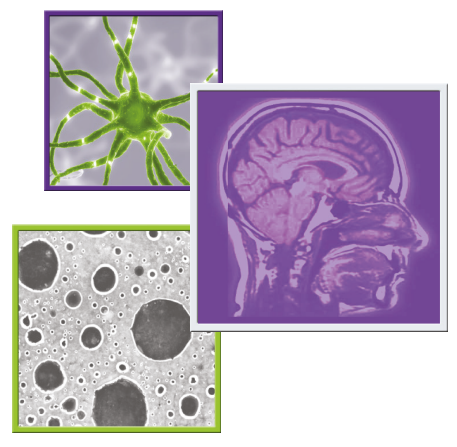

\author{
Hiba Alzoubi ${ }^{* 1,2}$ (D), Francesca Gianno ${ }^{2}$ (D) Felice Giangaspero ${ }^{2,3}$, Daniela Bartolini ${ }^{4}$ \\ Luca Riccioni ${ }^{4}$, Evelina Miele ${ }^{\ddagger}, 5$ \& Manila Antonelli ${ }^{\ddagger}, 2$ \\ ${ }^{1}$ Department of Basic Medical Sciences, Faculty of medicine, Yarmouk University, Irbid, Jordan \\ ${ }^{2}$ Department of Radiological, Oncological \& Anatomopathological Sciences, Sapienza University of Rome, Rome, Italy \\ ${ }^{3}$ IRCCS Neuromed-Mediterranean Neurological Institute, Pozzilli, Italy \\ ${ }^{4}$ Department of Pathology, Bufalini Hospital, Cesena, Italy \\ ${ }^{5}$ Department of Paediatric Haematology/Oncology, IRCCS Bambino Gesù Children's Hospital, Rome, Italy \\ *Author for correspondence: heba.z@yu.edu.jo \\ ${ }^{\ddagger}$ Authors contributed equally
}

Atypical teratoid/rhabdoid tumor (ATRT) is a malignant CNS embryonal tumor that mostly occurs in childhood, adult cases are rare. We report a case of a 23-year-old male with an extra-axial dura-based lesion in the left frontal area, previously diagnosed as gliosarcoma. After 6 years, the patient had a recurrence and the previous slides were reviewed. Tumor was positive for vimentin and negative for INI1. The differential diagnosis for this extra-axial tumor with long survival was rhabdoid meningioma with INI1 loss or ATRT. DNA methylation profiling was performed to reach the final and the most definitive diagnosis; the result was ATRT. Our case suggests the usefulness of DNA methylation profiling for diagnosing challenging CNS tumors.

First draft submitted: 24 March 2020; Accepted for publication: 22 April 2020; Published online: 30 June 2020

Keywords: atypical teratoid rhabdoid tumor $\bullet$ DNA methylation profiling • INI1 loss $\bullet$ rhabdoid meningioma

Atypical teratoid/rhabdoid tumor (ATRT) is a malignant CNS embryonal tumor composed of poorly differentiated elements, including rhabdoid cells, with inactivation of SMARCB1 (INI1) or rarely of SMARCA4 (BRG1) [1].

ATRT is the most common malignant brain tumor in children below the age of 6 months [2], it can be located in either supratentorial or infratentorial locations [1] and may occasionally develop in adolescents and adults [3]. In adults, ATRT affects predominantly females and is mostly located in the sellar region [4-6].

SMARCB1/INI1 is one of the core subunit proteins of the ATP-dependent-SWI/SNF chromatin remodeling complex [7,8]. SMARCB1/INI1 protein loss has been reported in various tumors in many organs [7,9]. The aberrant expression could be either complete, mosaic or reduced. Many tumors have a complete loss of INI1 in addition to ATRT of the brain, including malignant peripheral nerve sheath tumors and epithelioid sarcoma [7].

DNA methylation profiling is a highly robust and reproducible approach for the classification of CNS tumors. This technique exploits the concept that cancer methylome is a combination of somatically acquired DNA methylation changes and characteristics reflecting cell of origin and events contributing to transformation [10].

DNA methylation profiling of ATRT identified three molecular subgroups with specific transcriptional signatures: ATRT-SHH, ATRT-TYR and ATRT-MYC [11]. These subgroups are different in regard to the age of onset, the location of the tumor, SMARCB1 mutational pattern and radiological features [11,12]. We report the case of a duralbased frontal tumor in an adult male with histological and clinical features that suggest a diagnosis of rhabdoid meningioma with INI1 loss or ATRT. This led us to order DNA methylation profiling to reach the final and the most definitive diagnosis and the tumor was classified as ATRT.

Our case highlights the importance of DNA methylation profiling as a tool for diagnosis in selected adult cases that do not fulfill all the specific criteria for a specific tumor histotype. 

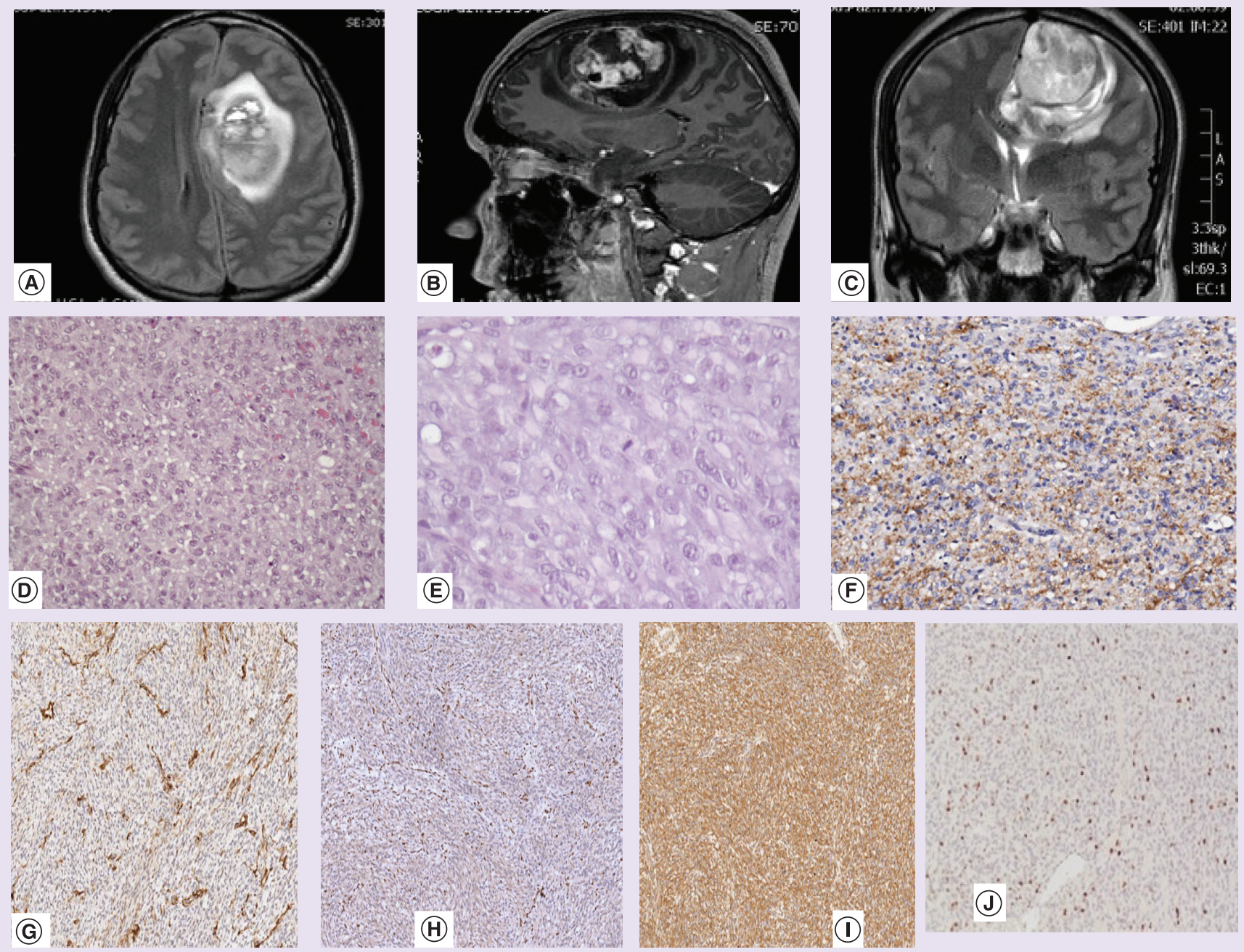

Figure 1. Radiological, histopathological and immunohistochemical features of the tumor. (A) FLAIR sequence MRI of the head showing hyperdense large tumor with midline shift and mass effect. (B) T1 sequence postcontrast and (C) and T2 sequence show a large lobulated heterogeneously enhancing extra-axial tumor, attached to dura. Histopathological examination shows (D) Highly cellular tumor composed of rhabdoid cells $(H \& E \times 100)$. (E) Rhabdoid cells show eccentric nuclei, prominent nucleoli and eosinophilic cytoplasm (H\&E 400). Immunohistochemical stains show (F) Focal positivity for synaptophysin $(\times 200)$ and (G) Smooth muscle actin ( $\times 200)$, (H) Loss of INI1 ( $\times 200)$, (I) Diffuse positivity for vimentin $(\times 200)$ and $(\mathrm{J}) \mathrm{Ki67}(\times 100)$. Negative markers including CK, GFAP and EMA (Not shown).

FLAIR: Fluid attenuation inversion recovery; H\&E: Hematoxylin and eosin stain; T1: Longitudinal relaxation time; T2: Transverse relaxation time.

\section{Case description}

A 23-year-old male presented with headache, cognitive-motor slowing and right motor hemi syndrome in 2013. MRI showed a large extra-axial dura-based lesion in the left frontal area with altered signal and focal contrast enhancement (Figure $1 \mathrm{~A}-\mathrm{C}$ ). The mass was diagnosed as gliosarcoma (WHO Grade IV). The patient was treated with adjuvant radio- and chemotherapy for 3 months and temozolomide for six cycles. In 2018 there was a suspected recurrence of the tumor, and MRI showed an altered signal area around the surgical cavity with a contrast enhancement nodule. In consideration of the long clinical history of a gliosarcoma, the original slides were reviewed.

\section{Methylation profiling}

DNA methylation profiling was performed according to protocols approved by the institutional review board after obtaining written consent from the patient. Tumor areas with the highest tumor cell content $(\geq 70 \%)$ were selected for DNA extraction. Samples were analyzed using Infinium-Human Methylation EPIC Bead Chip (Illumina, CA, 




Figure 2. Copy number variation profile analysis - depiction of structural rearrangements involving autosomes and X/Y chromosomes. Gains/amplifications represent positive (green), losses negative (red) deviations from the baseline. 29 tumor relevant genomic regions are highlighted. The red arrow indicates SMARCB1 focal deletion.

USA) arrays according to the manufacturer's instructions, as previously reported [13-15]. Generated methylation data were compared with the Heidelberg brain tumor classifier to assign a subgroup score for the tumor compared with 91 different brain tumor entities [10].

\section{Pathology \& molecular findings}

The tumor showed a highly cellular tumor composed of rhabdoid cells with an eccentric nucleus, prominent nucleolus and eosinophilic cytoplasm. The tumor was mitotically active 5-9 mitosis/10 high power field (HPF) but without necrosis or vascular proliferation. No meningothelial whorls or pseudoinclusions were seen. The tumor infiltrated the adjacent brain parenchyma (Figure 1D \& E).

The tumor cells were diffusely positive for vimentin (Figure 1I), focally positive for synaptophysin and SMA (Figure 1F \& G) and negative for CK, GFAP and EMA. All tumor cells showed a loss of nuclear SMARCB1/INI1 protein expression with retained immunoreactivity in vascular endothelial cells (Figure 1H). Ki67 index was variable between different areas ranging between 5 and 15\% (Figure 1J).

Based on the radiological and clinical information the differential diagnosis was rhabdoid meningioma with INI1 loss or ATRT. DNA methylation profiling was performed to reach the final and the most definitive diagnosis. The tumor had a score of 0.98 in the methylation class ATRT, subclass MYC. Copy number variation analysis showed a loss of SMARCB1 (Figure 2).

\section{Discussion}

The first adult case of ATRT was reported in 1992 [16], and since then many cases have been reported in the literature [3]. Females are more commonly affected than males and the intracranial location is more common than the spine [3]. The most common intracranial location is suprasellar (46\%) followed by cerebral hemispheres (32\%) [3]. In our case, the tumor is located in the extra-axial meningeal location, a location rarely reported in the literature $[17,18]$.

ATRT of the brain is a SMARCB1-deficient tumor. SMARCB1 (SWI/SNF-related, matrix-associated, an independent regulator of chromatin, subfamily B, member 1), located at 22q11.23, also known as INI1, is a tumor 
suppressor gene, that encodes a core subunit of the $S W I / S N F$ chromatin-remodeling complex [19]. Inactivation of both copies of SMARCB1 leads to the loss of nuclear expression of SMARCB1 protein in tumors cells, which can be easily identified by using immunohistochemistry [20]. SMARCB1-deficient tumors can be divided into two groups according to intracranial or extracranial location. The extracranial SMARCB1-deficient tumors mostly comprise soft tissue tumors, such as epithelioid sarcoma, some extra skeletal myxoid chondrosarcomas and some myoepithelial tumors [21]. The intracranial SMARCB1-deficient tumors include ATRTs, cribriform neuroepithelial tumor [21,22] and rare, poorly differentiated chordomas [23]. These intracranial SMARCB1-deficient tumors are rarely reported in meningeal locations $[1,17,18,23-27]$.

The diagnosis of ATRT is easily suspected in infants, but the differential diagnosis is wider in adults as it is in our case. The differential diagnosis of rhabdoid morphology could be epithelioid/rhabdoid glioblastoma, composite rhabdoid tumors, including carcinomas, melanomas, sarcomas and desmoplastic small round cell tumors which have secondary rhabdoid phenotype [28], germinomas, embryonal tumors and rhabdoid meningiomas. Although these mentioned and the parent tumors in composite ones are characterized by retained INI1 expression [28], there are two reported cases about SMARCB1-deficient meningeal tumors [21]. This was our reference toward our main differential diagnosis of rhabdoid meningioma, in addition to extra-axial location, rhabdoid morphology and long survival, but the tumor was negative for EMA which has suboptimal sensitivity and specificity and vary according to the grades or subtypes of meningioma and it can be negative in high-grade meningioma [29]. In addition, a high Ki67 index, increased mitotic activity and absence of meningothelial whorls can happen in some high-grade rhabdoid meningioma [29].

DNA methylation profiling is a powerful tool that aids in the diagnosis of challenging CNS tumors. The integration of methylation-based CNS tumor classification into the diagnosis has significant clinical benefit for patients with ambiguous CNS tumors and can directly affect patients' management, avoid unnecessary or unwanted treatment in some cases, allow us to offer the best care for the patients and avoid unnecessary health costs [10,30,31]. Pathologists rarely use it for ATRT diagnosis because they depend mainly on INI1 loss; however, in our case, the presence of these two reported cases of SMARCB1-deficient meningeal tumors [21], the unique location of the tumor, the long term survival of 6-years after diagnosis, the absence of EMA staining and the positivity for SMA and synaptophysin led us to order methylation profile to reach the final and the most definitive diagnosis and for further epigenetic subgrouping [11], especially that this patient was previously misdiagnosed with gliosarcoma.

DNA methylation profile for pediatric ATRT brain tumors, enhancer landscape and subgroup-specific transcriptional networks separate ATRT into three molecular subgroups: ATRT-SHH, ATRT-TYR and ATRT-MYC [11]and these subgroups are different in regard to the age of onset, the location of the tumor, SMARCB1 mutational pattern and radiological features [11,12]. The ATRT-TYR subgroup is strongly associated with an infratentorial location and ATRT-MYC with supratentorial location, while ATRT-SHH appears in both locations. The methylation profile for our case fits with methylation class ATRT, with epigenetic similarities to pediatric ATRTs of the ATRT-MYC subgroup and a similar profile to the other reported adult ATRT case in the sellar region [4].

ATRTs in adults generally have a poor prognosis, but long-term survival is possible after surgery and adjuvant chemotherapy and radiotherapy [32,33]. In a systematic review of literature, the overall survival was 23 months and the median time to progression was 5 months [34]. However, in addition to our case, three reported cases of adult ATRT did show survival of more than 3 years [34-36]. Interestingly, two of the three reported cases were initially misdiagnosed as high-grade glioma $[35,36]$.

We report for the first time a case of an adult meningeal frontal tumor with histological features of rhabdoid meningioma but further classified by DNA-methylation profiling as ATRT. This molecular approach allows us to better define tumors with peculiar histopathological characteristics.

\section{Author contributions}

Study conception and design was done by M Evelina and A Manila. Methodology by A Hiba and M Evelina. Original draft preparation was done by A Hiba. Editing and completing the draft were done by A Hiba, M Evelina, G Francesca, G Felice, B Daniela and R Luca. Final review was done by A Manila and M Evelina.

All authors provided a critical review of the manuscript for intellectual content and approved the final version of the manuscript. 
Financial \& competing interests disclosure

This research was partially supported by 'Il Fondo di Giò Onlus' and from 'Fondazione Italiana per la Lotta al Neuroblastoma Onlus'. The authors have no other relevant affiliations or financial involvement with any organization or entity with a financial interest in or financial conflict with the subject matter or materials discussed in the manuscript apart from those disclosed.

No writing assistance was utilized in the production of this manuscript. This case report received no external funding.

Ethical conduct of research

The authors state that they have obtained verbal and written informed consent from the patient/patients for the inclusion of their medical and treatment history within this case report.

\section{Open access}

This work is licensed under the Attribution-NonCommercial-NoDerivatives 4.0 Unported License. To view a copy of this license, visit http://creativecommons.org/licenses/by-nc-nd/4.0/

\section{Summary points}

- SMARCB1/INI1 protein loss has been reported in various tumors in many organs and is an important marker for the diagnosis of these tumors.

- Rhabdoid morphology and INI1 loss should be taken seriously, even in abnormal locations and different age groups.

- DNA methylation profiling is a highly robust and reproducible approach for the classification of CNS tumors.

- DNA methylation profiling can be used as a tool for diagnosis in selected adult cases.

- The integration of methylation-based CNS tumor classification into the diagnosis has a significant clinical benefit for patients with ambiguous CNS tumors.

- The integration of methylation-based CNS tumor classification into the diagnosis can directly affect patients' management.

\section{References}

Papers of special note have been highlighted as: $\bullet \bullet$ of considerable interest

1. WHO classification of tumours of the central nervous system (Revised 4th Edition). Louis DN, Ohgaki H, Wiestler OD, Cavenee WK (Eds). IARC, Lyon, France, 209-212 (2016).

2. Frühwald MC, Biegel JA, Bourdeaut F, Roberts CWM, Chi SN. Atypical teratoid/rhabdoid tumors-current concepts advances in biology, and potential future therapies. Neuro. Oncol. 18(6), 764-778 (2016).

3. Chan V, Marro A, Findlay JM, Schmitt LM, Das S. A systematic review of atypical teratoid rhabdoid tumor in adults. Front. Oncol. 8 , 567 (2018).

-• Reviews all atypical teratoid/rhabdoid tumors (ATRTs) in adults in the literature and makes a summary regarding male and female distributions and common locations which were useful in our case report.

4. Johann PD, Bens S, Oyen F et al. Sellar region atypical teratoid/rhabdoid tumors (ATRT) in adults display DNA methylation profles of the ATRT-MYC subgroup. Am. J. Surg. Pathol. 42(4), 506-511 (2018).

5. Nakata S, Nobusawa S, Hirose T et al. Sellar atypical teratoid/rhabdoid tumor (AT/RT): a clinicopathologically and genetically distinct variant of AT/RT. Am. J. Surg. Pathol. 41(7), 932-940 (2017).

6. Moretti C, Lupoi D, Spasaro F et al. Sella turcica atypical teratoid/rhabdoid tumor complicated with lung metastasis in an adult female. Clin. Med. Insights Case Rep. 6, 177-182 (2013).

7. Kohashi K, Oda Y. Oncogenic roles of SMARCB1/INI1 and its deficient tumors. Cancer Sci. 108(4), 547-552 (2017).

8. Hollmann TJ, Hornick JL. INI1-deficient tumors: diagnostic features and molecular genetics. Am. J. Surg. Pathol. 35(10), e47-e63 (2011).

9. Margol AS, Judkins AR. Pathology and diagnosis of SMARCB1-deficient tumors. Cancer Genet. 207(9), 358-364 (2014).

10. Capper D, Jones D, Sill M et al. DNA methylation-based classification of central nervous system tumours. Nature 555(7697), 469-474 (2018).

11. Johann PD, Erkek S, Zapatka M et al. Atypical teratoid/ rhabdoid tumors are comprised of three epigenetic subgroups with distinct enhancer landscapes. Cancer Cell 29(3), 379-393 (2016).

-. Highlights an important and new method for subgrouping for ATRT by using methylation profiling, with the discovery of these three new subgroups, that helped us in further subgrouping of ATRT as in our case. 
12. Nowak J, Nemes K, Hohm A et al. Magnetic resonance imaging surrogates of molecular subgroups in atypical teratoid/rhabdoid tumor. Neuro. Oncol. 20(12), 1672-1679 (2018).

13. Petruzzellis G, Alessi I, Colafati GS et al. Role of DNA methylation profile in diagnosing astroblastoma: a case report and literature review. Front. Genet. 10, 391 (2019).

14. Flex E, Martinelli S, Van Dijck A et al. Aberrant function of the C-terminal tail of HIST1H1E accelerates cellular senescence and causes premature aging. Am. J. Hum. Genet. 105(3), 493-508 (2019).

15. Ballabio C, Anderle M, Gianesello M et al. Modeling medulloblastoma in vivo and with human cerebellar organoids. Nat. Commun. 11(1), 583 (2020).

16. Horn M, Schlote W, Lerch KD, Steudel WI, Harms D, Thomas E. Malignant rhabdoid tumor: primary intracranial manifestations in an adult. Acta Neuropathol. 83(4), 445-448 (1992).

17. Taneja AK, Reis F, Zanardi VA, Rogerio F, Queiroz LS. Meningeal presentation of an atypical teratoid/rhabdoid tumor. Neurol. India 58(4), 681-682 (2010).

18. El-Nabbout B, Shbarou R, Glasier CM, Saad AG. Primary diffuse cerebral leptomeningeal atypical teratoid rhabdoid tumor: report of the first case. J. Neurooncol. 98(3), 431-434 (2010).

19. Wilson BG, Roberts CW. SWI/SNF nucleosome remodellers and cancer. Nat. Rev. Cancer 11(7), 481-492 (2011).

20. Hollmann TJ, Hornick JL. INI1-deficient tumors: diagnostic features and molecular genetics. Am. J. Surg. Pathol. 35(10), e47-e63 (2011).

21. Dadone B, Fontaine D, Mondot L et al. Meningeal SWI/SNF related, matrix-associated, actin-dependent regulator of chromatin, subfamily B member 1 (SMARCB1)-deficient tumours: an emerging group of meningeal tumours. Neuropathol. Appl. Neurobiol. 43(5), 433-449 (2017).

22. Hasselblatt M, Oyen F, Gesk S et al. Cribriform neuroepithelial tumor (CRINET): a nonrhabdoid ventricular tumor with INI1 loss and relatively favorable prognosis. J. Neuropathol. Exp. Neurol. 68(12), 1249-1255 (2009).

23. Renard C, Pissaloux D, Decouvelaere AV, Bourdeaut F, Ranchere D. Non-rhabdoid pediatric SMARCB1-deficient tumors: overlap between chordomas and malignant rhabdoid tumors? Cancer Genet. 207(9), 384-389 (2014).

24. Arnold MA, Stallings-Archer K, Marlin E et al. Cribriform neuroepithelial tumor arising in the lateral ventricle. Pediatr. Dev. Pathol. 16(4), 301-307 (2013).

25. Ibrahim GM, Huang A, Halliday W et al. Cribriform neuroepithelial tumour: novel clinicopathological, ultrastructural and cytogenetic findings. Acta Neuropathol. 122(4), 511-514 (2011).

26. Mobley BC, McKenney JK, Bangs CD et al. Loss of SMARCB1/INI1 expression in poorly differentiated chordomas. Acta Neuropathol. 120(6), 745-753 (2010).

27. Park JY, Kim E, Kim DW, Chang HW, Kim SP. Cribriform neuroepithelial tumor in the third ventricle: a case report and literature review. Neuropathology 32(5), 570-576 (2012).

28. Barresi V, Branca G, Raso A, Mascelli S, Caffo M, Tuccari G. Atypical teratoid rhabdoid tumor involving the nasal cavities and anterior skull base. Neuropathology 36(3), 283-289 (2015).

29. Perry A, Louis DN, Budka H et al. Meningioma. In: WHO classification of tumours of the central nervous system (Revised 4th Edition) (Chapter 10). Ohgaki H, Wiestler OD, Cavenee WK, Louis DN (Eds). IARC press, Lyon, France, 232-245 (2016).

30. Hsiao M-C, Holinka L, Peracchio M et al. Path-46. Detecting misdiagnosed atypical teratoid/rhabdoid tumor (ATRT) by DNA methylation-based tumor classification. Neuro. Oncol. 21(Suppl. 6), vi153-vi154 (2019).

31. Karimi S, Zuccato JA, Mamatjan Y et al. The central nervous system tumor methylation classifier changes neuro-oncology practice for challenging brain tumor diagnoses and directly impacts patient care. Clin. Epigenet. 11, 185 (2019).

32. Arita K, Sugiyama K, Sano T, Oka H. Atypical teratoid/rhabdoid tumour in sella turcica in an adult. Acta Neurochir. (Wien) 150(5), 491-495 (2008).

33. Buscariollo DL, Park HS, Roberts KB, Yu JB. Survival outcomes in atypical teratoid rhabdoid tumor for patients undergoing radiotherapy in a surveillance, epidemiology, and end results analysis. Cancer 118(17), 4212-4219 (2012).

34. Dardis C, Yeo J, Milton K et al. Atypical teratoid rhabdoid tumor: two case reports and an analysis of adult cases with implications for pathophysiology and treatment. Front. Neurol. 8, 247 (2017).

35. Makuria AT, Rushing EJ, McGrail KM, Hartmann DP, Azumi N, Ozdemirli M. Atypical teratoid rhabdoid tumor (AT/RT) in adults: review of four cases. J. Neurooncol. 88(3), 321-330 (2008).

36. Takahashi K, Nishihara H, Katoh M et al. Case of atypical teratoid/rhabdoid tumor in an adult, with long survival. Brain Tumor Pathol. 28(1), 71-76 (2011). 